\title{
Das Konzil von Chalkedon in der Darstellung des Liberatus von Karthago (Breviarium 11-14)
}

\author{
von Martin Wallraff
}

Der hier zu behandelnde Abschnitt im Breviarium des Liberatus (Kapitel 11-14) behandelt das Konzil von Chalkedon samt Vor- und einem kleinen Stück Nachgeschichte. Die insgesamt vier Kapitel sind unterschiedlicher Länge: das letzte ist durch seine Kürze und auch inhaltlich von den anderen abgesetzt. In den ersten drei Kapiteln (11, 12 und 13) wird von je einer Synode berichtet, nämlich von der endemischen Synode in Konstantinopel im Jahr 448, der "Räubersynode“ in Ephesus im Jahr 449 sowie der (später so genannten) ökumenischen Synode in Chalkedon im Jahr 451. Das 14. Kapitel berichtet vom Beginn der Chalkedon-Rezeption in Alexandria und ist mit dem Folgenden enger verknüpft als mit dem Vorausgehenden - es hätte also auch (und vielleicht besser) dem nachfolgenden Abschnitt zugewiesen werden können ${ }^{1}$.

Die Kapiteleinteilung ist stimmig und korrespondiert gut mit den Überschriften im Pinax, wie er in den Handschriften dem Text vorausgeht. Aus dieser Sicht steht der Vermutung nichts im Wege, dass das eine wie das andere (Einteilung und Überschriften) vom Autor selbst stammt.

Im Folgenden wird zunächst der Inhalt des Textes kurz referiert, gefolgt von wenigen Bemerkungen zur Überlieferung und Textkritik. Sodann wird die Quellenfrage diskutiert, und schließlich werden anhand ausgewählter Beobachtungen Tendenzen und Intentionen des Autors erhoben. Ausblickshaft werden Bemerkungen zur Wirkungsgeschichte am Schluss stehen. Revolutionäres wird dabei nicht zum Vorschein kommen. Natürlich tritt in den späteren Passagen das historiographische Profil des Autors viel deutlicher in Erscheinung als dort, wo er sich auf - noch dazu: bekannte - Quellen stützt.

\section{Inhalt}

Nach der Schilderung des Konfliktes um Ibas von Edessa im vorausgehenden Kapitel setzt Liberatus (Kapitel 11) recht unvermittelt und ohne direkten Zusammenhang mit der redaktionellen Floskel his temporibus

1 Vgl. dazu den Beitrag von Hanns-Christoph Brennecke im vorliegenden Band. 
neu ein. In Konstantinopel tritt der Presbyter und Abt Eutyches auf, der in seinen Predigten eine anstößige christologische Lehre vertritt. Euseb von Dorylaeum beschwert sich beim Patriarchen Flavian und fordert ihn auf, Eutyches vor die in Konstantinopel tagende endemische Synode zu ziehen, um sich ein Bild von seiner Häresie zu machen. Eutyches widersetzt sich mehreren Vorladungen und lässt schließlich seine hervorragenden Kontakte zum Kaiserhof, namentlich zu dem Eunuchen Chrysaphius spielen, um jedenfalls nicht allein, sondern in Begleitung zweier kaiserlicher Bevollmächtigter zu erscheinen. Vor der Synode teilt Eutyches mit, er könne die Aussage „aus zwei Naturen“ für Christus nur vor der Vereinigung gelten lassen, danach bekenne er eine Natur. Daraufhin verdammt ihn die Versammlung. Eutyches lässt die Dinge nicht auf sich beruhen, sondern setzt alle Hebel in Bewegung, um das Urteil rückgängig zu machen. Er wendet sich brieflich an den römischen Bischof Leo und veranlasst außerdem auf der politischen Ebene eine formale Prüfung seines Prozesses. Diese scheitert, bestätigt also die Verurteilung. Das Kapitel wird geschlossen durch das relativ unmotiviert eingeschobene Glaubensbekenntnis des Flavian.

Das Folgende (Kapitel 12) knüpft wiederum direkt an die EutychesGeschichte an und berichtet, wie der Abt und Presbyter nun auch den alexandrinischen Patriarchen einschaltet. Dioskur erwirkt bei Kaiser Theodosius II. die Einberufung einer allgemeinen Synode in Ephesus. Theodoret und Ibas werden am Erscheinen gehindert; Dioskur hingegen kommt mit schlagkräftiger Begleitung. Die Delegaten Roms werden brüskiert; im Anschluss wird Eutyches rehabilitiert, und die Synode erklärt seinen Glauben für korrekt. Die Bischöfe Theodoret von Cyrus und Ibas von Edessa werden in absentia verurteilt, ebenso Euseb von Dorylaeum; es folgt eine wahre Orgie von weiteren Verdammungen - fast karikaturhaft geschildert. Obgleich Flavian dabei nicht direkt genannt wird, folgt dessen Appell an den römischen Amtskollegen. Nach dem Ende der Synode sind in diversen Diözesen Nachfolgeregelungen erforderlich; dies ruft mancherorts ein Schisma hervor. Leo von Rom bemüht sich beim Kaiser in Konstantinopel vergeblich um eine Revision des Urteils und eine erneute ökumenische Synode.

Das längste Kapitel (13) behandelt das Konzil von Chalkedon. Dieses wird möglich durch den Tod Theodosius' II.; dessen Nachfolger Marcianus nimmt den Prozess wieder auf. Das ursprünglich für Nizäa in Aussicht genommene Treffen wird näher an die Hauptstadt verlegt, weil die römischen Delegaten die Anwesenheit des Kaisers wünschen. Im Folgenden wird jede der zwölf Sitzungen knapp zusammengefasst. In den ersten beiden Sitzungen werden wichtige vorausgehende Dokumente verlesen; in der dritten wird Dioskur in absentia verurteilt und abgesetzt. In der vierten Sitzung wird der Tomus Leonis als dogmatische Grundlage akzeptiert. In der fünften und sechsten wird dann doch eine eigene Glaubensformel abgefasst und in Anwesenheit des Kaisers feierlich approbiert. In der siebten und achten Sitzung werden unter anderem die Bischöfe Theodoret und Ibas 
rehabilitiert. Die folgenden drei Sitzungen sind konkreten Konfliktfragen gewidmet; in Abwesenheit der römischen Delegaten werden zum Schluss auch Privilegien für den Sitz von Konstantinopel beschlossen. Dagegen protestieren die Römer in der zwölften und letzten Sitzung, doch ohne jeden Erfolg.

Dass die Konsequenzen des Konzils vor allem für Alexandria gravierend waren, liegt auf der Hand: darum geht es im 14. Kapitel. Es gibt Schwierigkeiten bei der Besetzung der Nachfolge Dioskurs. Schließlich wird Proterius gewählt und geweiht, doch Timotheus Aelurus und Petrus Mongos organisieren den Widerstand und stellen sich an die Spitze der Partei Dioskurs.

\section{Zur Textgestalt}

Der Text ist durch Eduard Schwartz in einer leicht zugänglichen und sehr lesbaren Form hergestellt worden. In dem hier zu besprechenden Abschnitt gibt es sogar einige Fälle, in denen der Text allzu leicht oder genauer: allzu klassisch lesbar gemacht worden ist. Schwartz hat, wie es in der damaligen Philologengeneration üblich war, relativ intensiv eingegriffen, wo das in den Handschriften überlieferte Latein nicht unmittelbar verständlich war oder nicht seinen Vorstellungen von Korrektheit entsprach. Diese Eingriffe zeugen stets von großer Vertrautheit mit der Sache und mit der Sprache; sie sollten in jedem Falle als Vorschlag ernst genommen werden. Gleichwohl wird man in einzelnen Fällen auch zu abweichenden Urteilen kommen. Das betrifft zunächst grammatische Fragen. In frühmittelalterlichem Latein kann man ad abbatibus etwa durchaus tolerieren ${ }^{2}$. Auch die Differenzierung von Ordinal- und Kardinalzahlen muss nicht notwendigerweise mit klassischer Rigeur durchgeführt worden sein; in dem Ausdruck post septimum numerum dierum (Hss.) ist daher die Korrektur in septem vielleicht nicht zwingend $^{3}$. Wenn die Handschriften synodi Epheseni primi schreiben ${ }^{4}$, ist gleichfalls nicht sicher, ob ein Kopist synodus maskulin verstanden hat oder ob man diesen Lapsus nicht auch dem Autor Liberatus zutrauen will (Schwartz korrigiert zu synodi Ephesenae primae) ${ }^{5}$. In einzelnen Fällen löscht Schwartz ein pleonastisch zugefügtes Subjekt ${ }^{6}$, wo es sich aus dem Kontext ohnehin ergibt, doch kann die etwas umständliche Ausdrucksweise durchaus auch die des Autors sein. Nicht nötig ist auch spolians

2 Liberatus, Breviarium 11 (ACO II/5, 114,27 Schwartz); vgl. Peter Stotz, Handbuch zur lateinischen Sprache des Mittelalters, Bd. 4. Formenlehre, Syntax und Stilistik, HAW II/ 5,4, München 1998, 277-279 (\$29).

Liberatus, Breviarium 11 (114,32 S.).

Liberatus, Breviarium 13 (120,10 S.).

Vgl. Stotz (wie Anm. 2), 147f. (\$74.2).

Vgl. Liberatus, Breviarium 11 (114,10 S.): Eusebius; Breviarium 12 (117,27f. S.): Dioscurus. 
(Schwartz) statt expolians ${ }^{7}$ oder die Löschung von autem in Kapitel $13^{8}$. In Kapitel 11 kann man überlegen, ob man das überlieferte fide $e^{9}$ statt in sed (Schwartz) nicht lieber in fidei korrigieren sollte: das würde semantisch auch gut zum Folgenden passen.

Nur in wenigen Fällen haben solche textkritischen Diskussionen inhaltliche Konsequenzen. Einigermaßen weit reichend ist die Zufügung von non bei der in Konstantinopel tagenden Synode des Flavian ${ }^{10}$. Bevor man ein Wort wie non ohne Anhalt in den Handschriften ergänzt, sollte man alle anderen Möglichkeiten ausgelotet haben. In diesem Falle böte es sich an, wie die Zeugen IT zu verfahren und das in den älteren Handschriften PW überlieferte ideo wegzulassen. Es mag sein, dass IT ihrerseits hier nicht mehr als eine gelehrte Konjektur bieten, doch ist sie mindestens so gut wie die von Schwartz vorgeschlagene. Im Sinn ändert sich allerdings letzten Endes nicht allzu viel. Schwieriger ist die namentliche Nennung der römischen Delegaten Iulius und Hilarus bei der Synode $449^{11}$. Bei Hilarus erkennt der Leser wenigstens durch die Klammern im Text, dass der Name nicht überliefert ist, doch ein Blick in den Apparat zeigt, dass auch Iulius erst durch Schwartz' Korrektur in den Text gekommen ist. Dass die Ergänzung im Sinne des historischen Verlaufs sachlich berechtigt ist, steht außer Frage ${ }^{12}$, doch standen die Namen auch bei Liberatus? Es ließen sich verschiedene Heilungsversuche auf der Basis des (zugegeben problematischen) handschriftlichen Befundes denken, die weniger massiv in den Text eingreifen. Die Frage ist inhaltlich nicht komplett irrelevant, weil die (ohnehin von der Quelle nicht gedeckte) Nachricht im einen Fall das Missverhalten Dioskurs, im anderen Fall das Missverhalten gegenüber den römischen Delegaten stärker betont.

Solche Überlegungen sollten indes auf keinen Fall verdecken, dass viele von Schwartz' Eingriffen nötig oder sogar brillant sind: etwa die Änderung von igitur zu agitur ${ }^{13}$ und manch andere Fälle.

\section{Quellen}

Woher hat Liberatus seine Kenntnisse? Die Frage ist leicht zu beantworten, weil er selbst darüber Auskunft gibt und weil Eduard Schwartz in seiner Edition diese Auskunft geprüft und in die kleine Münze konkreter

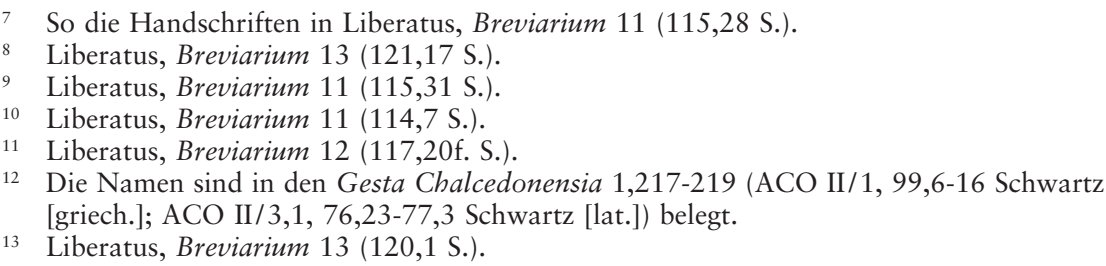


Apparateinträge umgerechnet hat ${ }^{14}$. Ex gestis synodalibus vel sanctorum patrum epistulis hoc breviarium collegi, so sagt Liberatus im Proömium seines Werkes ${ }^{15}$, und genau diese Auskunft trifft selbstredend primär für den Bericht der Synoden von 448, 449 und $451 \mathrm{zu}$ - alle drei sehr gut dokumentiert in den Konzilsakten von Chalkedon, die frühere jeweils inkorporiert in die spätere, weil ihre Akten auf der nachfolgenden Synode verlesen wurden ${ }^{16}$. Liberatus schreibt davon in der - soweit ich sehen kann - einzigen Notiz zu seinen Quellen außerhalb des Proöms:

„Doch für den, der genauer bescheid wissen will über die Räubereien Dioskurs, die er auf jener Synode vollbrachte: die erste Verhandlung der Synode von Chalkedon, bei der die Akten des zweiten Konzils von Ephesus (das indes nicht so genannt werden darf) verlesen und dem Protokoll beigefügt wurden, macht dem Leser alles vollkommen klar. Denn wir haben dies in der Übersetzung übernommen, die kürzlich in Alexandria aus dem Griechischen ins Lateinische angefertigt worden ist" ${ }^{\text {"17 }}$.

Diese Übersetzung ist unter den drei frühen lateinischen Fassungen mit der von Schwartz so genannten „versio antiqua correcta“, abgekürzt $\Phi^{c}$, zu identifizieren ${ }^{18}$.

Der Hauptgrund für diese Identifikation ist die (falsche) Position der Canones von Chalkedon: sie figurieren bei Liberatus ebenso wie in der Version $\Phi^{c}$ ganz am Ende des Konzilsgeschehens ${ }^{19}$, während die „versio antiqua " noch dem griechischen Original folgt und sie nach der sechsten Verhandlung einreiht. Außerdem zitiert er das Glaubensbekenntnis des Flavian, das aus der epistularum ante gesta collectio stammt, die in $\Phi^{a}$

14 Vgl. E. Schwartz, Praefatio, in: Concilium Universale Chalcedonense, edidit Eduardus Schwartz, ACO II/5. Collectio Sangermanensis, Berlin/Leipzig 1936, (V-XXII) XVIII sowie die jeweiligen Apparateinträge.

15 Liberatus, Breviarium 1 (99,1f. S.).

16 Für einen ersten Überblick über die reiche (aber komplexe) Überlieferungslage vgl. A. Grillmeier, Jesus Christus im Glauben der Kirche, Bd. II/1. Das Konzil von Chalcedon (451). Rezeption und Widerspruch (451-518), Freiburg 1986, 24-32. Weiterhin ist die synoptische Zusammenstellung der griechischen und lateinischen Textzeugen von Rudolf Schieffer, in: ACO IV/3,1, 502-512 von großem Nutzen.

17 Liberatus, Breviarium 12 (119,5-8 S.): Sed quaerenti plenius scire latrocinia Dioscuri in ipsa synodo perpetrata prima actio Chalcedonensi synodi, in qua nec nominandi Ephesini secundi concilii recitata sunt acta gestis inserta, legenti omnia plenissime innotescit. nam nos ista nuper Alexandriae de Graeco in Latinum translata suscepimus.

18 Vgl. Schwartz, Praefatio (wie Anm. 14), XVIII. Der Text ist (ebenfalls von Schwartz) ediert in ACO II/3 (im Apparat sind jeweils die Versionen $\Phi^{\mathrm{a}}$, $\Phi^{\mathrm{c}}$ und $\Phi^{\mathrm{r}}$ kenntlich gemacht), zur Überlieferung und zu den Unterschieden der Versionen vgl. E. Schwartz, Praefatio, in: Concilium Universale Chalcedonense, edidit Eduardus Schwartz, ACO II/3: Epistularum ante gesta collectio. Actio prima, Berlin/Leipzig 1935, (V-XVIII) VII-XV.

19 Liberatus, Breviarium 13 (123,8-12 S.). Vgl. E. Schwartz, Aus den Akten des Concils von Chalkedon, ABAW.PPH 32/2, München 1925, 17f. Auch die Nummerierung der Sitzungen folgt $\Phi^{c}$, nicht der älteren Version $\Phi^{a}$ (vgl. Liberatus, Breviarium 13 [122,21 S.]). 
noch nicht enthalten war $^{20}$. Andererseits kann Liberatus auch nicht mit der im Jahr 564 angefertigten Version des Rusticus gearbeitet haben, wie sich aus diversen Detailbeobachtungen am Text ergibt ${ }^{21}$. Man mag eine gewisse Versuchung verspüren, die Ergänzungen zu den Konzilsakten mit dem ansonsten rätselhaften Graecum Alexandriae scriptum des Proöms zu identifizieren, doch bei näherem Zusehen legt sich diese Identifikation nicht nahe ${ }^{22}$. Berührungen mit vorhandenen Kurzbeschreibungen der Synode von Chalkedon sind nicht nachzuweisen ${ }^{23}$. Die zuvor genannten gesta synodalia vel sanctorum patrum epistulae decken im Übrigen das Vorhandene ausreichend ab. Während die „versio antiqua“ in Konstantinopel erstellt wurde, könnte die hier verwendete korrigierte Version theoretisch durchaus aus Alexandria stammen - besondere Plausibilität oder zusätzliche Indizien über Liberatus hinaus sprechen allerdings nicht für diese Hypothese ${ }^{24}$.

Wie man dem Apparat von Schwartz entnehmen kann, ist Liberatus' Text zu sehr großen Teilen durch die Informationen aus den Konzilsakten erklärbar. Mit dieser Feststellung ist indes noch nicht allzu viel gewonnen, denn von den Akten - selbst in ihrer übersetzten Form - bis zu der knappen Darstellung des Breviarium ist der Weg dennoch relativ weit. Aus der

20 Vgl. Schwartz, Praefatio (wie Anm. 14), XVIII; das Glaubensbekenntnis steht in Liberatus, Breviarium 11 (116,17-117,3 S.).

21 In Breviarium 11 (115,5 S.) liest Liberatus mit $\Phi^{\text {ac }}$ sanctarum ecclesiarum et catholicae fidei, dagegen $\Phi^{\mathrm{r}}$ et de sanctis dei ecclesiis et pro catholica fide (Gesta Chalcedonensia 1,468 [ACO II/3,1, 120,26f. Schwartz]). In Breviarium 11 (116,5 S.) liest Liberatus mit $\Phi^{\text {ac }}$ quae, dagegen $\Phi^{\mathrm{r}}$ quos (Gesta Chalcedonensia 1,575 [137,2 S.]). Siehe weitere Fälle unten in Anm. 28 und 30.

22 Vgl. Liberatus, Breviarium 1 (99,3 S.). Der rote Faden der Ereignisse stammt aus den Konzilsakten, wie einige wörtliche Übereinstimmungen zeigen. Eine weitere durchlaufende schriftliche Quelle ist nicht zu erkennen. Für punktuelle Ergänzungen lägen die wörtlichen Zitate von Urkunden nahe, doch gerade diese stammen aus der lateinischen Übersetzung. Die Wendung illa quae in Graeco Alexandriae scripto accepi (Liberatus, Breviarium 1 [99,2f. S.]) muss im Übrigen nicht zwingend auf eine griechische Schrift zielen, die in Alexandria abgefasst wurde, sondern könnte auch dasjenige meinen, „das ich in griechischer Schrift in Alexandria empfangen habe" - damit wäre Alexandria nur der Ort, an dem Liberatus den Text kennen gelernt hat, nicht der Ort der Abfassung.

23 Einen Bericht ähnlichen, etwas größeren Umfangs hat der Kirchenhistoriker Theodorus Lector Anfang des 6. Jh.s abgefasst, ebenfalls auf der Basis der Konzilsakten. Dieser Text ist erst nach Abschluss der Edition (Theodoros Anagnostes, Kirchengeschichte, hg. von G.C. Hansen, GCS N.F. 3, Berlin $\left.{ }^{2} 1995\right)$ zum Vorschein gekommen und jetzt ediert von G.C. Hansen, Ein kurzer Bericht über das Konzil von Chalkedon, in: L. Burgmann (Hg.), Fontes Minores X, FBRG 22, Frankfurt 1998, 101-139. Berührungen mit Liberatus sind

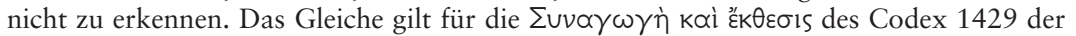
Athener Nationalbibliothek (fol. 4r-27v), deren Edition W. Brandes vorbereitet. Er hat dankenswerterweise bei der Tübinger Tagung auf den Text hingewiesen und eine provisorische Abschrift zugänglich gemacht. Auch mit dem Synodicon Vetus (Synodicon Vetus, ed. J. Duffy/J. Parker, CFHB.W 15, Washington D.C. 1979) sind keine Berührungspunkte festzustellen. Dass in den diversen weiteren Konzilsüberblicken, die in der byzantinischen Literatur kursierten, Liberatus-Parallelen zu finden sind, ist nicht völlig ausgeschlossen, doch sehr unwahrscheinlich.

24 Mit einiger Not könnte man auch hier den oben in Anm. 17 gegebenen Text so deuten, dass Alexandria nicht der Ort der Übersetzung, sondern der Bekanntschaft mit dem Text ist. 
indigesta moles der weitläufigen Verhandlungen von Chalkedon hatte der Historiograph ein schlüssiges und vor allem chronologisch angeordnetes Bild der Ereignisse herauszupräparieren, das auch ohne begleitende Aktenlektüre verständlich sein und Wesentliches von Unwesentlichem trennen sollte.

Gewiss, er konnte als Leitfaden eine knappe Zusammenfassung der Ereignisse verwenden, wie sie ihm etwa bei Prosper Tiro vorlag (und wie sie von ihm auch stellenweise benutzt worden sein $\mathrm{mag}^{25}$ ). Aufs Ganze gesehen ist er seiner Aufgabe aber sehr selbständig und insgesamt recht überzeugend gerecht geworden. Dies möge vorausgeschickt werden, wenn nun im Einzelnen zum Verständnis der Tendenzen und Intentionen natürlich gerade solche Punkte herausgepickt werden, an denen Liberatus Dinge nicht oder falsch verstanden hat oder Akzente gesetzt, die aus großem zeitlichen Abstand eher problematisch und fernliegend erscheinen.

\section{Tendenzen und Intentionen}

Um das spezifische Profil des Autors herauszuarbeiten, seien zunächst ungeordnet und unverbunden einige Beobachtungen gesammelt, die sich aus der Betrachtung des Textes, teilweise im Vergleich zu seinen Quellen, ergeben. Die erste Beobachtung ist besonders auffällig, wenn man aus einer wissenschaftlichen Perspektive kommt, die Theologiegeschichte stark betont, vor allem aus der Sicht klassischer (protestantischer) Dogmengeschichte der Alten Kirche etwa Harnackschen Typs. Dieser Zweig der Chalkedon-Rezeption stellt die Definition einer neuen christologischen Bekenntnisformel in den Mittelpunkt, und genau davon erfährt Liberatus' Leser eigentlich fast nichts: mit Mühe und Not die Tatsache, dass es ein solches symbolum überhaupt gegeben hat und dass es bei der fünften Sitzung mit großer Mehrheit akklamiert und in Geltung gesetzt worden is $^{26}$. Über den Inhalt verliert unser Autor kein Wort, er scheint ihn auch nicht besonders zu interessieren. Man muss diesen Befund aber nicht als theologisches Desinteresse deuten, sondern kann annehmen, dass Liberatus der Meinung war, der sicher auch viele Konzilsteilnehmer waren, dass nämlich über die klassischen Bekenntnistexte von Nizäa und Konstantinopel sowie die nun allgemein anerkannten brieflichen Äußerungen von Cyrill von Alexandria und Leo von Rom hinaus eine weitere Festlegung schlicht nicht nötig war. Schon gar nicht auf Druck des Kaisers: diesen lässt Liberatus recht bescheiden auftreten und nur seine Rolle als Bekräftiger des Glaubens betonen, ganz im Anschluss an den verehrten Konstantin. Machtpolitik spiele keine Rolle, so erklärt er vor der versammelten Synode,

25 Vgl. Liberatus, Breviarium 2 (99,18-20 S.); es geht dort um die Nestorius-Affäre.

26 Vgl. Liberatus, Breviarium 13 (121,34f. S.): Dictatum est fidei symbolum cum definitione et ab omnibus confirmatum est. 
non ad potentiam ostendendam sei er gekommen ${ }^{27}$. Wer möchte, kann aktuelle Bezüge dabei heraushören. Und dass es der Kaiser war, der auf der Erarbeitung einer neuen Bekenntnisformel in Chalkedon gedrungen hatte, wird auch Liberatus nicht entgangen sein.

Freilich ist auch sonst der Bericht über die Synoden von Ephesus 449 und Chalkedon 451 weitgehend theologie-frei. Ein Leser, der nur diese Abschnitte läse, hätte keine Vorstellung von den christologischen Streitfragen, die verhandelt wurden, bzw. er käme kaum auf die Idee, dass es überhaupt um Theologie ging. Doch auch hier liegt die Erklärung weder darin, dass Liberatus davon nichts wusste oder wissen wollte, noch und erst recht nicht darin, dass er inhaltliche Reserven gegenüber der theologischen Linie hatte, die sich letztlich durchsetzte. Vielmehr ist für ihn der Kern der Debatte die Kontroverse um Eutyches, und in dem dafür einschlägigen Kapitel finden sich sehr wohl theologische Aussagen, allerdings mehr ex negativa parte. Man erfährt also mehr über die verurteilte Christologie des umstrittenen Mönches als über orthodoxe Lehre. Der Angeklagte ließ sich nach einigem Hin und Her vor der endemischen Synode zu folgender Aussage drängen (die genügte, um ihn zu verurteilen):

„Er sagte, er habe die Meinung des heiligen Cyrill und des Athanasius auf seiner Seite, da sie sagten, dass er [Christus] zwar aus zwei Naturen vor der Vereinigung sei, doch nach der Vereinigung und Fleischwerdung nicht mehr zwei, sondern eine Natur habe“"28.

Diese Kernaussage hat Liberatus aus den Akten übernommen. Eine ähnliche Passage findet sich schon ganz zu Beginn des Abschnitts ${ }^{29}$. Für die positive Entfaltung der Zwei-Naturen-Lehre zitiert er hingegen eigenartigerweise nicht den Tomus Leonis, der in Chalkedon rezipiert werden und die Basis für die folgende Entwicklung bilden sollte, sondern vielmehr das Glaubensbekenntnis des Flavian, das ziemlich unmotiviert und ungeschickt in den Lauf der Ereignisse eingeflochten wird ${ }^{30}$. Gewiss, auch dort erfährt

27 Vgl. Liberatus, Breviarium 13 (122,8 S.), aus Gesta Chalcedonensia 6,5 (ACO II/3,2, 411,1-15 Schwartz).

28 Liberatus, Breviarium 11 (115,23-25 S.): Ille autem dixit legem se habere sancti Cyrilli et Athanasii quoniam ex duabus quidem naturis dixerunt ante adunationem, post adunationem autem et incarnationem non duas naturas, sed unam; vgl. Gesta Chalcedonensia 1,542 (ACO II/3,1, 127,21-23 S.). Der Textvergleich im Einzelnen ist von Interesse. Die merkwürdige Wendung legem se habere (vielleicht gemeint: lectum se habere?) ist offensichtlich ein Missverständnis von ego legi, das auf der Basis des griechischen Textes ( $₹ \gamma \gamma \dot{\omega}$ åvé $\gamma v \omega v$ - ACO II/1, 144,18-20 S.) nicht entstehen könnte. Die Version des Rusticus ergänzt nach incarnationem non das Wort iam, das in $\Phi^{\mathrm{c}}$ wie auch bei Liberatus fehlt.

29 Vgl. Liberatus, Breviarium 11 (114,2f. S.).

30 Nach der Bestätigung der Verurteilung des Eutyches (von Flavian ist nicht unmittelbar die Rede) schließt zeitlich und logisch schwer verständlich an: Postea expetivit imperator a Flaviano fidei confessionem [...], das Bekenntnis dann Breviarium 11 (116,17-117,3 S.), entnommen nicht aus den Konzilsakten, sondern aus der epistularum ante gesta collectio 1 (ACO II/3,1, 5,1-30 S.), die in der versio antiqua nicht enthalten war. Einige Details im Text lassen auch hier den Schluss zu, dass Liberatus mit $\Phi^{c}$, nicht mit Rusticus 
man von dem Kern der Lehre, und man sieht, wie der gemäßigten Mittelpartei der Ausdruck „aus zwei Naturen“ durch die Inanspruchnahme des Eutyches entwunden worden ist. Dennoch kann man sich des Eindrucks nicht erwehren, dass Liberatus keinen besonderen Wert auf die Rolle Roms legt und insbesondere nicht an der Feststellung interessiert ist, die weitere Geschichte sei die Geschichte der Durchsetzung der Linie und Theologie Roms. Selbst in der Kürzestfassung des Prosper Tiro wird dieser Eindruck erweckt $^{31}$. Liberatus hingegen paraphrasiert den Tomus des Papstes in zwei Zeilen: „Leo aber richtete ein Schreiben an Flavian, das die gesunde Lehre von der Inkarnation Christi enthielt und in wunderbarer Weise die Behauptungen des Eutyches zunichte machte." ${ }^{32}$

Von einigem Interesse ist der Abschnitt über die vergeblichen Bemühungen Leos, beim Kaiser eine erneute Konzilsverhandlung zu erlangen. Diesen Abschnitt hat Liberatus aus den Originaldokumenten (Briefen) selbst zusammengestellt und dabei den Kaiser Theodosius II. samt seinem korrupten Eunuchen Chrysaphius erstaunlich schonend behandelt. Gewiss erscheint Leo als fortissimus ${ }^{33}$, und Theodosius macht eine schlechte Figur, doch darf er unkommentiert sein Handeln auf den Wunsch nach pax et concordia in ecclesia zurückführen ${ }^{34}$.

Nicht weiter auffällig ist sodann die Beobachtung, dass Liberatus' Berichterstattung durchweg an der Dioskur-kritischen Haltung partizipiert, die für das gesamte Überlieferungsbündel von Anfang an charakteristisch ist. Die Schilderung des latrocinium (statt iudicium, um den Ausdruck Leos des Großen zu gebrauchen ${ }^{35}$ ) von Ephesus 449 gerät zur Karikatur. Bei der Rehabilitierung des Eutyches trägt der sonst so nüchterne Liberatus dick auf und gestaltet die Szene dramatisch aus (auch über die Quelle hinaus). Dioskur akzeptiert nicht nur dessen Lehre, sondern macht sie sich sogleich zu eigen und deutet sie als orthodoxen Ausdruck der Lehre der

gearbeitet hat: Breviarium 11 (116,34 S.) liest Liberatus mit $\Phi^{c}$ ex ambabus unus gegen ex ambobus Rusticus. Breviarium 11 (116,38 S.) kennt die Liberatus-Überlieferung die Variante sapientes quae eius sunt et loquentes (nämlich in den Hss. IT, danach wäre ein Punkt zu setzen), ähnlich $\Phi^{c}$; dagegen bei Rusticus sapientes quae eius ac loquentes ea et (dann weiter ohne Punkt, so allerdings auch in den Hss. PW bei Liberatus - am ehesten mit Kontamination zu erklären); der Editor hätte erwägen können, hier den Hss. IT zu folgen.

31 Vgl. Prosp., chron. 1358-1362.1369 (MGH.AA 9 = Chronica Minora 1, 480-482 Mommsen).

32 Liberatus, Breviarium 11 (115,32-34 S.): Leo vero direxit Flaviano tomum sanam incarnationis Christi doctrinam continentem et mirifice perimentem Eutychetis adsertiones.

33 Liberatus, Breviarium 12 (119,15 S.).

34 Liberatus, Breviarium 12 (119,25 S.).

35 Vgl. Leo M., ep. 95 ad Pulcheriam (20. Juli 451), in: Collectio Grimanica Nr. 51 (ACO II/4, 51,4 Schwartz): in illo Epheseno non iudicio, sed latrocinio. Leo nahm dabei übrigens eine Formulierung von Cicero (Cic., S.Rosc. 61) auf. Der Ausdruck latrocinium machte schnell die Runde und wird auch von Liberatus gebraucht: Breviarium 12 (119,5 S.); 13 $(121,13 f$. S.). Das „Räuberkonzil“ figuriert bekanntlich bis heute unter diesem Namen in den Handbüchern. 
Väter ${ }^{36}$. Mit den römischen Delegaten kam es zum Eklat, weil Dioskur ihren Anspruch auf den Vorsitz nicht akzeptierte und darüber hinaus das Schreiben von Papst Leo nicht verlesen lassen wollte ${ }^{37}$. Es folgt eine lange Serie von Verurteilungen. Immerhin gibt dieser Vorgang für Liberatus die willkommene schwarze Folie ab, auf der auch die Verurteilung Theodorets von Cyrus und des Ibas von Edessa erscheint: Beide werden unter skandalösen Umständen in absentia verdammt. Dabei stört es nicht, dass ausweislich der syrisch erhaltenen Konzilsakten von einer Vorladung des Ibas keine Rede sein kann ${ }^{38}$. Denn je skandalöser die Verurteilung, desto berechtigter erscheint die spätere Rehabilitierung beider Bischöfe auf dem Konzil von Chalkedon. Sie erscheint dringend geboten, ja geradezu als natürliche Konsequenz des Vorausgehenden. Dass auch hier aktuelle Bezüge eine Rolle spielen, wird man annehmen dürfen: Wer die beiden antiochenischen Theologen wiederum verurteilt, stellt sich gegen Chalkedon und damit auf die Seite Dioskurs.

Von großem Interesse ist weiterhin der Umgang mit dem umstrittenen Konzilsbeschluss über die Privilegien Konstantinopels, also dem später so gezählten canon 28 von Chalkedon. Dass der ganze Vorgang bei Liberatus erst am Ende der Konzilstätigkeit erscheint, hängt mit seiner Quelle zusammen: schon dort waren die Canones in diese Position gelangt. In der vorletzten Sitzung werden in einer zweiten Verhandlung „nach Abgang der Richter und Senatoren und der Delegaten des apostolischen Stuhls" einige Privilegien für den Sitz von Konstantinopel beschlossen ${ }^{39}$. Warum die genannten staatlichen und römischen Würdenträger nicht anwesend waren, wird nicht weiter motiviert. Jedenfalls blieb den Römern nichts weiter übrig, als am nächsten Tag in der letzten Sitzung gegen die Beschlüsse zu protestieren. Die Versammlung nahm davon Kenntnis, doch „, von allen Richtern und Bischöfen ist dieser Einspruch nicht angenommen worden“. Und - so fügt Liberatus lakonisch hinzu: „Wenn auch der apostolische Stuhl bis heute dem widerspricht, was von der Synode unterschrieben worden ist, so bleibt es doch durch den Schutz des Kaisers einstweilen bestehen" 40 . Eine dezidiert pro-römische Berichterstattung sähe anders aus.

36 Liberatus, Breviarium 12 (117,25-30 S.). Schwartz führt im Apparat einige der Stellen aus den Konzilsakten auf, an denen diese Aussage nicht steht (aber stehen müsste, wenn sie intendiert wäre).

37 Liberatus, Breviarium 12 (117,20-25 S.), siehe auch zur Textkritik oben bei Anm. 12. Nur der zweite Sachverhalt ist von den Konzilsakten gedeckt, vgl. Gesta Chalcedonensia 1,217-227 (ACO II/3,1, 76,23-78,28 S.).

38 Liberatus, Breviarium 12 (117,31-118,2 S.), vgl.: Akten der Ephesinischen Synode vom Jahre 449. Syrisch, hg. von J. Flemming, übersetzt von G. Hoffmann, Berlin 1917, 2-5.

39 Vgl. Liberatus, Breviarium 13 (123,9 S.): Post discessum iudicum et senatorum et legatorum apostolicae sedis.

40 Vgl. Liberatus, Breviarium 13 (123,16-18 S.): A iudicibus et episcopis omnibus illa contradictio suscepta non est. et licet sedes apostolica nunc usque contradicat, quod a synodo firmatum est, imperatoris patrocinio permanet quoad modo. 
Dennoch kann aufs Ganze gesehen festgehalten werden, dass Liberatus unvoreingenommen, zuverlässig und verständlich von den Vorgängen um Chalkedon berichtet. In seiner nüchternen Art ist es ihm gelungen, ein Bild zu entwerfen, das seinen Quellen gegenüber nur gelegentlich und in geringem Umfang überzeichnet und karikiert. Theologisch ist sein Interesse stärker auf die Häresie als auf die Orthodoxie gerichtet. Kirchenpolitisch ist der Nordafrikaner Liberatus der seltene Fall eines lateinischen Schriftstellers, der ohne besondere Sympathie für Rom schreibt.

\title{
5. Zur Nachwirkung
}

Es mag sein, dass die Reserve Rom gegenüber dazu beigetragen hat, dass das Breviarium zwar im lateinischen Westen nie ganz in Vergessenheit geriet (die vier Handschriften, auf die sich Schwartz stützt, bezeugen es), doch andererseits auch nie den Schritt in den engeren Kanon historischer Grundkenntnisse getan hat. Auf der Basis von Euseb und Hieronymus (der Chronik wohlgemerkt) formte sich so etwas wie eine Kette basaler Geschichtsüberlieferung, die das lateinische Mittelalter formen sollte. In dieser Kette kam die Rolle, über Chalkedon zu berichten, Prosper Tiro zu. Damit waren freilich die konkreten Kenntnisse über das Konzil sehr beschränkt, denn dort umfasst der Bericht zu der gesamten Ereignisfolge nur knapp drei Seiten ${ }^{41}$. Auf dieser Basis war es schwer, sich ein angemessenes Bild auch und gerade von den theologischen Streitpunkten zu machen. Martin Luther hat daher nicht ganz Unrecht, wenn er 1539 in „Von den Konziliis und Kirchen“ schreibt:

\begin{abstract}
„Was aber die Ursache dieses Konzilii sei gewesen, das wollt ich selbst gern von einem anderen lernen, denn hierher reicht keine glaubwürdige Historia. Die Ecclesiastica [scil. Euseb] höret auf mit dem ersten Konzilio Nicäno, die Tripartit und Theodoretus mit dem dritten zu Ephesus, und müssen nun fort fast des Papsts und der Seinen Historien glauben, welchen sehr mißlich ist zu glauben" "42.
\end{abstract}

Indes war Besserung in Sicht, denn ein Jahr vor Luthers Konzilienschrift war die erste große neuzeitliche Konziliensammlung erschienen, nämlich die des Kölner Franziskaners Peter Crabbe: „Concilia omnia, tam generalia quam particularia" 43 . Darin waren zum ersten Mal auch ausführlichere Informationen über Chalkedon greifbar - und zwar nicht zuletzt eben durch unseren karthagischen Diakon Liberatus (für den Crabbes Sammlung die editio princeps war $\left.^{44}\right)$. Luther hatte die Arbeit noch vor Abschluss seines

41 Siehe Anm. oben 31.

42 Luther, Von den Konziliis und Kirchen (WA 50, 592f.).

43 P. Crabbe: Concilia omnia, tam generalia quam particularia, 2 Bde., Köln 1538 (VD 16: C5644).

44 Die Konzilsakten in Bd. 1, fol. CCCXCIII ${ }^{\mathrm{r}}$ bis fol. DXII ${ }^{\mathrm{r}}$ (endend mit einem interessanten Kolophon, das über die handschriftliche Grundlage Auskunft gibt); Liberatus in Bd. 2, fol. LI $^{\mathrm{r}}$ bis fol. LXII ${ }^{\mathrm{v}}$ (der Abschnitt zu Chalkedon, Kapitel 11-14, fol. LV ${ }^{\mathrm{r}}$ bis fol. LVIII'). 
Buches zur Kenntnis genommen: sie habe, sagt er, „alle Konzilia in ein Buch zusammengebracht, welche Arbeit mir nicht übel gefället" "45. Was er indes über Chalkedon schreibt, macht nicht den Eindruck, als habe er sich sehr ausführlich mit der neuen Publikation auseinandergesetzt. Die Beschreibung der Theologie des Eutyches ist mehr erraten als fundiert ( „Ich will meine Gedanken sagen, treff ichs, wohl, wo nicht, so ist hiemit der christliche Glaube nicht gefallen "46). Immerhin beruft er sich an zwei Stellen auf die „Historien und auch die acta conciliorum “47: Damit meinte er die lateinische Übersetzung der Konzilsakten durch Rusticus und die Zusammenfassung durch Liberatus.

Auch das Verfasserteam der Magdeburger Zenturien war weitgehend auf dieselbe Materialbasis angewiesen. Freilich war unterdessen auch die Kirchengeschichte des Evagrius publiziert worden (zuerst griechisch 1544, dann auch in lateinischer Übersetzung durch Wolfgang Musculus 154948). Die Zenturiatoren kannten und zitierten alle drei bei der Darstellung des Konzils von Chalkedon: Rusticus, Liberatus und Evagrius. Am Schluss kommen auch sie auf die umstrittenen Privilegien des Bischofssitzes von Konstantinopel zu sprechen. Hier lassen sie es sich nicht nehmen, das Sätzchen des Liberatus zu zitieren, in dem er vom vergeblichen Widerspruch der Römer berichtet und seine Auffassung über die Gültigkeit des Beschlusses kundtut ${ }^{49}$. So fand die kleine Stichelei des Liberatus Eingang in die Kontroverstheologie der Reformationszeit.

Die historischen Studien, die nicht zuletzt durch die Reformation ausgelöst waren, führten schließlich zur Publikation auch der Konzilsakten in ihrer Originalsprache in der editio Romana 1608-1612 ${ }^{50}$. Danach erlosch jedoch das Interesse an Liberatus weitgehend - bis zu Eduard Schwartz und zu der Tübinger Tagung des Jahres 2009.

45 Luther, Von den Konziliis und Kirchen (WA 50, 514).

46 Luther, Von den Konziliis und Kirchen (WA 50, 595).

47 Luther, Von den Konziliis und Kirchen (WA 50, 598, siehe auch 600).

48 Der griechische Text ist in der großen Sammelausgabe der Kirchenhistoriker von Robert

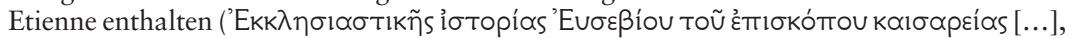
Paris 1544). Dieses gesamte Textcorpus wurde von Wolfgang Musculus in lateinischer Übersetzung vorgelegt (Ecclesiasticae historiae autores Eusebij Pamphili Casarice [...], Basel 1549 [VD 16: E4278]). Vgl. zu beiden Werken und zu weiteren Einzelheiten M. Wallraff, Die Rezeption der spätantiken Kirchengeschichtswerke im 16. Jahrhundert, in: L. Grane, A. Schindler und M. Wriedt (Hgg.), Auctoritas Patrum II. New Contributions on the Reception of the Church Fathers in the $15^{\text {th }}$ and $16^{\text {th }}$ Century, VIEG Beiheft 44, Mainz 1998, 223-260.

49 Ecclesiastica Historia integram ecclesiae Christi ideam [...] perspicuo ordine complectens congesta per aliquot studiosos et pios viros in urbe Magdeburgica, 13 Zenturien in 11 Bänden, Basel 1559-1574, hier Zenturie 5 = Bd. 3, 949,30-38.

50 Concilia generalia ecclesiae catholicae, 4 Bde., Rom 1608-1612, vgl. dazu H.J. Sieben, Die katholische Konzilsidee von der Reformation bis zur Aufklärung, Paderborn 1988, 231-234. 
Anhang: Das Konzil von Chalkedon.

Gliederung von Liberatus, Breviarium 11-14

Kapitel 11: Der Prozess des Eutyches

113,33-114,12 Auftreten und Lehre des Eutyches; Versuch des Euseb von Dorylaeum, ihn bei Flavian anzuschwärzen; sein Auftritt vor der endemischen Synode

114,12-32 Vergebliche Versuche, Eutyches vorzuladen

114,32-115,11 Eutyches versichert sich der Begleitung zweier kaiserlicher Beamter (Magnos und Florentius), kurzes kaiserliches Schreiben zur Bevollmächtigung des Florentius

115,12-29 Verhandlung vor der Synode, Verurteilung des Eutyches

115,29-35 Schreiben des Eutyches an Leo von Rom und Schreiben Leos an Flavian

115,35-116,6 Eutyches erwirkt beim Kaiser erneute Prüfung des Prozesses

116,7-13 Bestätigung der Akten der Verurteilung

116,14-117,3 Glaubensbekenntnis des Flavian

Kapitel 12: Die „Räubersynode“ von Ephesus 449

117,4-16 Eutyches beschwert sich bei Dioskur von Alexandria; dieser erwirkt beim Kaiser (Theodosius II.) ein ökumenisches Konzil; der Kaiser beruft es nach Ephesus ein (ohne Teilnahme des Theodoret und des Ibas)

117,16-30 Durchführung des Konzils mit Eutyches und Flavian als Angeklagten; Ausschaltung der römischen Delegaten; Bekräftigung der Lehre des Eutyches

117,31-118,9 Verurteilung des Ibas und des Theodoret in absentia

118,10-16 Verurteilung des Euseb von Dorylaeum; einige Bischöfe bitten für Flavian

118,17-23 Absetzung weiterer Bischöfe

118,24-28 Abschluss des Konzils; Flavian appelliert an Rom

118,29-119,4 Neubesetzung einiger Bischofsstühle, Schisma zwischen den Parteien

119,5-8 Redaktionelle Bemerkung zu den Quellen: Verweis auf Akten von Chalkedon

119,8-29 Vergeblicher Versuch Leos, bei Theodosius II. eine erneute Synode zu erwirken

Kapitel 13: Das Konzil von Chalkedon

119,30-120,7 Wiederaufnahme der Angelegenheit durch Markianos; Einberufung eines neuen Konzils, erst nach Nizäa, dann nach Chalkedon; erneute Untersuchung der Vorgänge

120,8-14 Sitzung 1: Verlesung der Akten von Ephesus I und II, Akklamation der Bischöfe gegen Dioskur 
120,15-24 Sitzung 2: Verlesung von zwei Briefen Cyrills und des Tomus Leonis

120,25-121,9 Sitzung 3: Vorladung des Dioskur; seine Verurteilung in absentia

121,10-31 Sitzung 4: Verhandlung über das Geschick weiterer Bischöfe; Glaubensbekenntnis im Anschluss an den Tomus Leonis; Schwierigkeiten mit der Lage in Ägypten

121,32-122,5 Sitzung 5: Formulierung eines eigenen Glaubenssymbols im Anschluss an Anatolius von Konstantinopel; es soll dem Kaiser vorgelegt werden

122,6-12 Sitzung 6: Auftritt des Kaisers vor dem Konzil; feierliche Verabschiedung des Symbols

122,13-20 Sitzung 7: Klärung der Aufteilung der Patriarchate von Antiochia und Jerusalem; Rehabilitierung des Theodoret; Vertagung der Angelegenheit des Ibas

122,21-29 Sitzung 8: Klärung diverser Streitfragen; Rehabilitierung des Ibas; Nichtanerkennung von Ephesus II als Synode

122,30-123,4 Sitzung 9 und 10: Absetzung zweier Bischöfe; Klärung eines Streites zwischen den Sitzen von Nizäa und Nikomedien

123,5-12 Sitzung 11: Wiedereinsetzung eines Bischofs; Verabschiedung einiger Canones und von Privilegien für den Sitz von Konstantinopel (in Abwesenheit der apostolischen Legaten!)

123,12-18 Sitzung 12: Protest der apostolischen Legaten, doch wird der Beschluss für gültig erklärt

Kapitel 14: Streitigkeiten in Alexandria

123,19-33 Exilierung Dioskurs; Wahl des Proterius als Bischof von Alexandria

123,33-124,5 Schisma in Alexandria, Timotheus Aelurus und Petrus Mongos führen die Partei des Dioskur

\section{ABSTRACT}

The analysis of Breviarium 11-14 raises various questions, e.g. why the theology of Chalcedon is nearly missing. Neither the Chalcedonense nor the Tomus Leonis are quoted. Only the position of Eutyches and that of Flavian become clear. Thus the tendency of the Breviarium seems to be sceptical towards interpretations according to which the Christological debate can be seen as victory of a clear position of the bishops of Rome. Even the canons of Chalcedon (with canon 28) are located on a wrong place in the narrative order, but here Liberatus follows only his sources. 